\title{
Open Access und die Novellierung des deutschen Urheberrechts
}

\author{
Andreas Degkwitz \\ Brandenburgische Technische Universität Cottbus \\ Informations-, Kommunikations- und Medienzentrum \\ degkwitz@tu-cottbus.de
}

\begin{abstract}
Wissenstransfer erweist sich für Hochschulen und Universitäten als Schlüsselfaktor und steht in unmittelbarem Zusammenhang mit der wissenschaftlichen Informationsversorgung. Die traditionelle (papiergebundene) Publikationspraxis kann die digitale Wissensversorgung erheblich beeinträchtigen, weil sie auf einer Wertschöpfungskette beruht, der Produktionsverfahren für gedruckte Publikationen zugrunde liegen. Dies hat eine Rollenverteilung in der Wertschöpfungskette zur Folge, durch die Autoren ihre Verbreitungs- und Verwertungsrechte (als Bestandteile ihres Urheberrechts) an Verlage abtreten, die den Herstellungs- und Verbreitungsprozess für Monographien und Zeitschriften übernehmen und damit das wirtschaftliche Risiko für den Verkauf dieser Produkte tragen. Mit dieser Rollenverteilung verbinden sich vor allem dann gute Voraussetzungen für Gewinn- und Umsatzmaximierung durch Preissteigerungen, wenn das Produktportfolio einen marktbeherrschenden Monopolcharakter hat. Insbesondere bei Zeitschriften der naturwissenschaftlichen, technologieorientierten und medizinischen Fachgebiete hat sich diese Entwicklung seit langem eingestellt. Die Folge ist ein - aufgrund hoher Abonnementkosten - eingeschränkter Zugang sowie ein fast ausschließlich kommerziell getriebenes Distributions- und Marktverhalten.
\end{abstract}

Knowledge transfer is obviously a key factor for colleges and universities and is directly associated with the provision of scientific information. The traditional (paper-based) process of publication can significantly impair digital provision of knowledge because it rests upon a chain of added value that is based on the production process for printed publications. It leads, in turn, to a division of roles in the chain of added value through which authors relinquish their rights of distribution and utilization (as elements of copyright) to publishing houses, which undertake the production and distribution of monographs and journals and herewith the economic risks involved with the sale of these products. This allocation of roles is associated with high chances for the maximization of profit and sales volume through price increases in as far as a product portfolio occupies a monopolistic market position. This has long been the case in particular for journals in the fields of science, technology and medicine. The result - due to high subscription costs - is limited access and an almost exclusively commercially-oriented system of distribution and marketing.

Neue Medien- und Internettechnologie bieten vergleichsweise einfache, schnelle Produktionsoptionen, globale und rasche Distributionsmöglichkeiten sowie orts- und zeitunabhängige Zugänglichkeit. Um diese neuen Möglichkeiten für Produktion und Distribution von Veröffentlichungen besser zu nutzen und um zugleich der Monopolisierung des wissenschaftlichen Publikationsmarktes entgegenzuwirken, wurden neue Modelle der Herstellung, Verbreitung und Vermittlung von Wissensobjekten im Sinne von Open Access entwickelt und umgesetzt. Ausgangspunkt dafür ist, dass das kostenintensive Original einer Publikation mit allen Rechten des Autors elektronisch vorliegt und mit Hilfe verfügbarer Technologie (Internet) distribuiert und damit veröffentlicht werden kann. Grundsätzlich können Autoren oder Rechteinhaber selbst ihre Publikationen über das Internet verbreiten, ohne dabei auf einen Verlag angewiesen zu sein.

Auf dieser Basis ist eine Neuverteilung der Rollen und Rechte der Akteure der Wertschöpfungskette des wissenschaftlichen Publizierens möglich - mit der Folge, dass sich neue Geschäfts- und Organisationsmodelle für den Informations- und Wissenstransfer umsetzen lassen und die verfügbare Technologie von der Wissenschaft selbst für eine rasche, weltweite Bereitstellung ihrer Veröffentlichungen 
eingesetzt werden kann. Universitäten und Hochschulen können in diesem Kontext eine sehr viel aktivere Rolle als bisher übernehmen, indem sie den Publikationsprozess stärker beeinflussen und nach ihren (wissenschaftseigenen) Konditionen steuern. Unter solchen Rahmenbedingungen sind die Autoren nicht mehr in der Situation, ihre Verbreitungs- und Verwertungsrechte zwingend abtreten zu müssen. Vielmehr können Forschung und Lehre die ,Hoheit' über ihre eigene Produktion behalten, so dass grundsätzlich die Voraussetzung gegeben ist, den Wissenstransfer und die Informationsversorgung weniger ,marktabhängig' zu gestalten. Entscheidend für die Umsetzung neuer Publikationsformen ist allerdings deren Akzeptanz durch die Wissenschaftler (als Autoren und Nutzer). In diesem Zusammenhang spielt Rechtssicherheit eine zentrale Rolle, so dass sich entsprechende Verträge mit Autoren oder Herausgebern im Interesse aller Beteiligten auch im Open-Access-Kontext empfehlen.

\section{Aktuelle Entwicklungen der deutschen Urheberrechtgesetzgebung}

Das Urheberrechtsgesetz bietet die rechtlichen Rahmenbedingungen, um die Rechte von Autoren und Verwertern vor missbräuchlicher Nutzung zu schützen. Wesentlich dabei ist ein tragfähiger Ausgleich zwischen den Interessen von Autoren und Verwertern einerseits sowie Nutzern und Rezipienten andererseits. Für den Bereich der akademischen Forschung und Lehre ist insofern eine besondere Situation gegeben, als die Gruppe der Autoren und die der Nutzer - im Vergleich zu Herstellern und Nutzern von Produkten des Konsumgütermarktes - einen hohen Übereinstimmungsgrad haben: Autoren sind Nutzer, um (als Autoren) wieder zu publizieren. Hinzu kommt, dass Bildung und Wissenschaft mit öffentlichen Geldern fi-

\section{Degkwitz: Open Access und die Novellierung des deutschen Urheberrechts. $\quad$ S. 244 ZfBB 54 (2007) 4-5, S.243-245.}

nanziert werden und sich insofern die Publikation von Forschungsergebnissen oder Lehrmaterialien gleichsam als öffentliches Gut interpretieren lässt - ein durchaus nachvollziehbares Verständnis, das jedoch durch das Urheberrecht so ausdrücklich nicht vorgegeben wird. Im Gegensatz dazu steht eine eindeutig gewinngetriebene Verwertung von wissenschaftlichen Datenbanken und Zeitschriften, die insbesondere bei den großen Verlagsmonopolen zu beobachten ist. Nicht zuletzt diese Monopole, die seit vielen Jahrzehnten steigende Gewinne und Umsätze verbuchen, dringen auf einen stärkeren Schutz der Verwertungsrechte, die - als autorenseitig abgetretene Rechte - zum Urheberrecht gehören.

Die begonnene Novellierung der Urheberrechtsgesetzgebung, die vor dem Hintergrund einer Vereinheitlichung des Urheberrechts in den europäischen Partnerländern auf der Grundlage der EURichtlinie 2001/29/EG erfolgte, berührt die Bereitstellung und Nutzung urheberrechtlich oder verwertungsrechtlich geschützter Materialien in Forschung und Lehre; dabei geht es vorrangig um folgende Paragraphen:

§ 31: Die Übertragung von Verwertungsrechten an Verlage für künftige (derzeit noch unbekannte) Nutzungsarten;

$\S 52 \mathrm{a}$ : Die Bereitstellung und Nutzung digitaler Materialien im (teilöffentlichen) Kontext von Lehrveranstaltungen oder Forschungsprojekten;

$\S 52 \mathrm{~b}$ : Die Begrenzung von ,on the spot consultations' auf definierte Arbeitsplätze der Bibliotheken;

$\S 53$ : Die digitale Privatkopie und damit verbundene technische Schutzmaßnahmen;

$\S 53 \mathrm{a}$ : Die Zulassung des Versandes elektronischer Kopien im Rahmen von Fernleih- und Dokumentlieferdiensten.

Die nun anstehende Novellierung der genannten Paragraphen, die der vorliegende Regierungsentwurf (März 2006) vorsieht und die zugleich nur einen Ausschnitt der Urheberrechtsgesetzgebung darstellt, lässt gegenüber der bisherigen Gesetzespraxis für Bildung und Wissenschaft erhebliche Einschränkungen bei der Bereitstellung und Nutzung von Literatur und Informationsressourcen privatwirtschaftlicher Anbieter erwarten. Die bis dato bestehenden Ausnahmeregelungen (Schranken) für Bildung und Wissenschaft sind im Fall einer Umsetzung des Regierungsentwurfs weitgehend aufgehoben. Leitend für die Gesetzgebung ist allem Anschein nach der Schutz der Verwerterrechte, indem bestehende ,Freiräume' eingeschränkt und die Nutzungspraxis in Forschung und Lehre an den (für den ConsumerMarkt üblichen) Rechtsrahmen angepasst werden sollen. Vor diesem Hintergrund sind Bestrebungen 
einer nachhaltigen Umsetzung von Open Access durchaus auch als Reaktion auf die sich für Bildung und Wissenschaft verschärfenden Rahmenbedingungen der Urheberrechtsgesetzgebung zu betrachten.

\section{Open Access und Urheberrecht}

Die Erwartungen, die rechtliche Position der Nutzer im Zuge der aktuellen Urheberrechtsnovellierung nachhaltig zu verbessern, waren und sind auf Seiten der Universitäten und Wissenschaftseinrichtungen hoch. Stimuliert wird dies durch die nachvollziehbaren und verständlichen Forderungen des „Aktionsbündnisses für Urheberrecht in Bildung und Wissenschaft". Zugleich führen die vielversprechenden Ansätze von Open Access zu der Einschätzung, dass sich die traditionellen Verlage in einer zunehmend schwachen Situation befinden, weil ihnen das Verlagsgeschäft von Bibliotheks- und Informationseinrichtungen mehr und mehr abgenommen wird. Dabei wird allerdings oft vergessen, dass der Marktanteil kommerzieller Zeitschriftenanbieter noch immer bei etwa $90 \%$ liegt und sich die Marktpenetration von Open Access noch in der Startphase befindet. Viele Wissenschaftler wollen weiterhin die Möglichkeit nutzen, in einer renommierten Zeitschrift zu publizieren, was ihnen auf Basis des Urheberrechts in keiner Weise versagt werden kann.

Ein ebenfalls häufiges Missverständnis betrifft den Gegenstand der rechtlichen Auseinandersetzung, der sich zum einen auf die Urheberrechtsgesetzgebung insgesamt und zum anderen wesentlich auf die oben genannten Paragraphen konzentriert. Beim Urheberrecht geht es um den rechtlichen Schutz derer, die als Autoren, Distributoren und Verwerter an der Veröffentlichung und Verbreitung geschützter Inhalte beteiligt sind. Der Streit zwischen Bibliotheken, Informationseinrichtungen und Verlagen im Zuge des Novellierungsprozesses bezieht sich primär auf verwertungsrechtlich geschützte Inhalte (Monographien und Zeitschriften kommerzieller Anbieter). Dabei ist die Urheberrechtsgesetzgebung in eine Vielzahl von Rechtsnormen eingebunden, die in Teilen auf der nationalen Ebene, z. T. auf der europäischen Ebene lokalisiert sind; gerade die EU-Richtlinien üben einen starken Druck auf die nationale Gesetzgebung aus.

In Anbetracht dessen muss deutlich gesagt werden, dass das Urheberrechtsgesetz keinerlei Möglichkeiten bietet, eine bestimmte Publikationspraxis für Forschung und Lehre vorzuschreiben. Die bisweilen geäußerte Meinung, in der Urheberrechtsgesetzgebung Open Access verankern zu können, gilt nicht für die im Rahmen der Urheberrechtsgesetzgebung vereinbarten Ausnahmeregelungen. Zudem wird genuines Open-Access-Publizieren durch die oben genann-

\section{Degkwitz: Open Access und die Novellierung des deutschen Urheberrechts. S. 245 ZfBB 54 (2007) 4-5, S.243-245.}

ten Paragraphen grundsätzlich nicht behindert; für die sog. Post-Print-Speicherung ist dies zumindest in Teilen differenzierter zu sehen (z. B. im Kontext von § 31).

Die fortschreitende Entwicklung des Open-Access- Publizierens könnte weiterhin zu der Annahme führen, dass ein Urheberrechtsgesetz eigentlich nicht mehr erforderlich sei und sich mit dem kostenfreien Zugang zu Open-Access-Publikationen im Grunde erübrige. Zu dieser Einschätzung ist eindeutig zu bemerken, dass das Urheberrecht und der damit verbundene Schutz der Autoren auch bei Open Access seine Gültigkeit hat. „Zu keiner Zeit verzichtet der Urheber bei einer Open-AccessVeröffentlichung oder -Zugänglichmachung auf seinen Urheberrechtsschutz. Indem er das Werk als Open-Access zur Verfügung stellt, übt er seine Verwertungsrechte aus. Er bestimmt, dass die Nutzung durch jedermann unter Wahrung der Persönlichkeitsrechte erfolgen kann.“ (Beger, S. 54) Demnach beruhen die kostenfreie Nutzung von Open-Access-Publikationen und der Verzicht auf eine kommerzielle Verwertung oder Verbreitung auf der Entscheidung des Autors, indem er von dieser Option (als Teil seines Urheberrechts) keinen Gebrauch macht. Insofern sind auch bei Open-Access-Publikationen Autorenverträge und/oder Lizenzierungsmodelle wie beispielsweise die Creative-Commons-Lizenz für den Schutz der Autoren (Rechtssicherheit) unbedingt ratsam.

Zusammenfassend lässt sich festhalten: Auch wenn sich Open-Access-Publizieren durchaus als Reaktion auf die aktuelle Novellierung der angesprochenen Paragraphen im deutschen Urheberrechtsgesetz versteht, besteht zwischen Open Access und Urheberrechtsgesetzgebung kein grundsätzlicher Widerspruch - im Gegenteil: Urheberrechtliche Regelungen gehören zu den zentralen Voraussetzungen für das wissenschaftliche Publizieren; dies gilt auch für Open Access. Mit dem Schutz der Autoren, den 
das Urheberrecht bietet, ist im Open-Access-Kontext der rechtliche Spielraum der Autoren - möglicherweise stärker als bisher - angesprochen, so dass sie von ihren Rechten in einer Weise Gebrauch machen können, dass ein freier Zugang zu Wissensobjekten ermöglicht wird und sich der Wissenstransfer damit weniger gewinn- und umsatzorientiert gestaltet.

\section{Literatur}

Beger, Gabriele: Urheberrecht für Bibliothekare. Eine Handreichung von A - Z. - Verlag Medien und Recht, München, 2006, 136 S.

Degkwitz, Andreas; Andermann, Heike: Zirkulation wissenschaftlicher Information in elektronischen Räumen. In: Wissen und Eigentum. Geschichte, Recht und Ökonomie stoffloser Güter, hrsg. von Jeanette Hofmann. - Bundeszentrale für Politische Bildung, Schriftenreihe, Band 552, Bonn, 2006, S. 221 - 240. www.bpb.de/publikationen/TRRZ2E,0,Wissen_und_Eigentum.html. [Stand 5.6.2007]

Göttinger Erklärung des Aktionsbündnisses ,Urheberrecht für Bildung Wissenschaft'. www. urheberrechtsbuendnis.de. [Stand 15.05.2007]

Hilty, Reto: Das Urheberrecht und der Wissenschaftler. In: Gewerblicher Rechtschutz und Urheberrecht Internationaler Teil. In: Zeitschrift der Deutschen Vereinigung für gewerblichen Rechtsschutz und Urheberrecht 3 (2006), S. 179 - 190.

Kuhlen, Rainer: Wissen als Eigentum. Wie kann der freie Zugang zu den Ressourcen des Wissens in globalen Informationsräumen gesichert werden? www.Wissensgesellschaft.org/themen/ publicdomain/wisseneigentum.pdf. [Stand 7.8.2003] 